\title{
Comportement des bourgeons de Pseudotsuga menziesii (Mirb) Franco cultivés en conditions contrôlées
}

\author{
Françoise PIVERT, Monique JACQUES, M. BONNET-MASIMBERT * \\ et E. MIGINIAC \\ CNRS, Institut de Physiologie végétale, F 91190 Gif-sur-Yvette \\ * INRA, Station d'Amélioration des Arbres forestiers, Ardon, F 45160 Olivet
}

\begin{abstract}
Résumé
Des Douglas issus de boutures âgées de 9 à 12 mois ont été soumis à divers traitements lumineux et thermiques afin d'observer la capacité de débourrement de l'ensemble des bourgeons. En début d'expérience, tous les bourgeons étaient en repos apparent. Par rapport à une photopériode courte $(9 \mathrm{~h})$ un éclairement de $16 \mathrm{~h}\left(250 \mu \mathrm{Em} \mathrm{m}^{-} \mathrm{s}^{-1}\right)$ stimule fortement le débourrement des bourgeons. Cette stimulation s'observe également pour un éclairement faible $\left(40 \mu \mathrm{E} \mathrm{m}^{-{ }^{-}} \mathrm{s}^{-1}\right)$, donné en complément de la période de $9 \mathrm{~h}$. Ces résultats ont été confirmés chez des plants issus de semis et âgés de 4 ans où, de plus, un éclairement pendant $16 \mathrm{~h}$ accroît fortement le nombre de bourgeons réactivés. Une période de froid $\left(5{ }^{\circ} \mathrm{C}\right.$ ) réactive tous les bourgeons d'une bouture si le traitement a duré 9 semaines. L'allongement de la pousse de première génération qui se produit dès la fin de la période froide est d'autant plus important que lc froid a duré plus longtemps. Enfin, des températures élevées données en alternance ( $35^{\circ} \mathrm{C}$ pendant $9 \mathrm{~h}$ et $25^{\circ} \mathrm{C}$ pendant $15 \mathrm{~h}$ ) durant 2 semaines accroissent notablement le débourrement des bourgeons. I et 3 semaines de ce traitement stimulent également, mais de façon moindre, le débourrement. Ces effets sont obtenus en régime d'alimentation hydrique et minérale non limitante. La discussion met l'accent sur l'hétérogénéité physiologique de la population de bourgeons d'un jeune plant révélée par des exigences différentes pour leur débourrement, en photopériodes longues ou en températures froides ou en températures chaudes.
\end{abstract}

\section{Introduction}

Le cycle végétatif d'un végétal ligneux adapté aux conditions climatiques des régions tempérées présente des alternances de phase d'activité et de repos. La période de dormance hivernale en est une des caractéristiques essentielles (Romberger, 1963). On admet classiquement qu'une période prolongée de froid (environ $+5^{\circ} \mathrm{C}$ ) lève la dormance (RıTchie, 1984) faisant passer progressivement un bourgeon de l'état de dormance à l'état de quiescence. Cet état est caractérisé par un certain besoin en températures élevées pour obtenir le début de la croissance (Romberger, 1963; Perry, 1971). Cependant, d'autres facteurs, comme la photopériode, l'intensité at la qualité de l'éclairement peuvent intervenir à ce moment et modifier profondément la croissance des bourgeons. C'est, en particulier, ce qu'ont observé Dormı.jng et al. 
(1968) sur le Picea abies à qui on peut faire effectuer 3 à 4 cycles par an selon les conditions du Phytotron de Stockholm. En faisant varier photopériode et température, il apparaît, à la suite de leurs travaux, qu'un passage au froid ne serait pas indispensable au débourrement à condition que la maturation des bourgeons ait eu lieu en conditions optimales, telles que : jour court et température de $20^{\circ} \mathrm{C}$.

Chez le Pseudotsuga menziesii, Allen \& Owens (1972) décrivent en détail les caractéristiques morphologiques des phases du développement. Des travattx postérieurs ont mis en évidence le rôle du froid pour lever la dormance (Rıtchul, 1984) de jeunes arbres et Campbeli. \& Sugano (1975) ont montré que d'autres facteurs comme la photopériode et des températures $>12^{\circ} \mathrm{C}$ pouvaient interférer avec l'effet du froid dans le développement de cette plante. Nous avons complété certaines de ces expériences grâce aux conditions très précises du Phytotron de Gif-sur-Yvette où étaient placés de jeunes arbres afin de savoir si le froid est indispensable à la levée de dormance de tous les bourgeons et si d'autres facteurs, comme la photopériode et la valeur de l'éclairement, pouvaient également exercer un rôle sur le débourrement de bourgeons écailleux.

Après une période de croissance, un végétal ligneux présente le plus souvent une phase de repos. Ainsi apparaît une rythmicité de croissance. Cette rythmicité classiquement observée (CHAMPAGNat, 1983), même en conditions contrôlées et constantes comme dans le cas du Corylus avellana (Barnola et al., 1977) permet de s’interroger également sur les causes de l'arrêt de croissance et de la formation de bourgeons écailleux qui l'accompagne. Dans la nature, le Pséudotsuga menziesii peut présenter une seconde vague de croissance, généralement dans le courant de l'été (BonNET-MASIMBERT, 1982). On peut penser que certaines conditions du milieu (forts éclairements et/ou températures élevées) associées à une rupture d'une phase de sécheresse provoquent cette nouvelle vague de croissance qui, dans ce cas, a lieu après formation d'un bourgeon écailleux. On a alors une pousse d'été qui, comme le propose JABLANCZY (1971), se distingue d'une croissance pratiquement libre ou continue telle qu'on peut parfois l'observer si toutes les conditions externes sont favorables.

De nombreuses questions apparaissent donc concernant le déterminisme écologique du cycle de développement du Pseudotsuga menziesii. Nous allons chercher à préciser les circonstances de la reprise de croissance de ces bourgeons ainsi que celles de l'arrêt de croissance ultérieur en cas de manifestation d'un rythme de croissance en conditions contrôlées. C'est pourquoi de jeunes plantes de Pseudotsuga menziesii, issues de boutures de manière à contrôler la variabilité génotypique, ont été placées en diverses situations d'environnement après que la croissance de tous les bourgeons ait été arrêtée par un séjour préalable en photopériode brève et température moyenne.

\section{Matériel et méthodes}

\subsection{Matériel}

Les essais concernent essentiellement des plants issus de boutures âgées de 9 à 12 mois. Seul un essai porte sur des boutures âgées de 4 ans. Les pieds-mères, 
sélectionnés par I'INRA pour la tardiveté du débourrement et la vigueur dans un test de descendances de peuplements artificiels français, sont 3 clones, âgés de 9 ans lors du prélèvement des boutures et taillés en haie depuis 3 ans. Bouturés fin mars 1984 selon la technique de ConNu (1973), les plants ont été rempotés fin août 1984 et transférés de l'INRA au Phytotron fin octobre 1984.

Chaque essai porte sur 3 à 5 boutures par clone et par modalité, sauf pour l'expérience portant sur l'influence de la photopériode, faite sur des plants âgés de 4 ans, avec un individu par condition.

\subsection{Conditions de culture}

Nous utilisons les conditions caractéristiques du Phytotron décrites par DE BiLderling \& Lourtioux (1976).

Les boutures sont faites sur un mélange constitué pour $1 / 3$ de tourbe et $2 / 3$ de pouzzolane et installées en serre sous mist. Au sevrage elles sont placées sur terreau d'écorces compostées (Fertil-Aquitaine). Elles sont ensuite repiquées sur vermiculite dès leur arrivée au Phytotron. Elles bénéficient de l'arrosage automatique par tubes capillaires. Ces plants sont placés 1 mois à $17^{\circ} \mathrm{C}$ en $9 \mathrm{~h}$ d'éclairement, puis 7 semaines à $12^{\circ} \mathrm{C}$ en $12 \mathrm{~h}$, avant la répartition dans les diverses conditions expérimentales. Ces séjours préalables ont assuré un bon enracinement des plants dans leur nouveau substrat et ont permis l'arrêt de croissance et la formation de bourgeons écailleux chez tous les plants. Ces plants mesurent environ $20 \mathrm{~cm}$. Leur axe principal est constitué, outre le fragment de rameau de la bouture initiale, de 1 à 2 générations de pousses plus rarement 3. Sur cet axe sont insérés de 0 à 2 rameaux axillaires. Les expériences ayant été conduites simultanément, les plantes sont donc toutes dans le même état initial. La température est contrôlée et constante à 22 "C pour la plupart des expériences. Pour quelques expériences, les températures suivantes ont été utilisées : $5^{\circ} \mathrm{C}, 35^{\circ} \mathrm{C} / 25^{\circ} \mathrm{C}\left(35^{\circ} \mathrm{C}\right.$ pendant $9 \mathrm{~h}$ et $25^{\circ} \mathrm{C}$ pendant $15 \mathrm{~h}$ par $\left.24 \mathrm{~h}\right)$. L'humidité relative de l'air varie d'une condition à l'autre, mais elle est toujours supérieure à 50 p. 100 , souvent égale à 70 p. 100 .

L'éclairement est mesuré à l'aide d'un quantamètre LI-COR (LAMBDA Instruments Corporation). Pour les radiations photosynthétiquement actives, entre 400 et $700 \mathrm{~nm}$, il est de $250, \mathrm{LE} \mathrm{m}^{-2} \mathrm{~s}^{-1}$ quand il est fourni par des tubes fluorescents (MAZDA 65, T 12, Blanc industrie) avec un appoint d'incandescence. Dans certains cas un éclairement incandescent faiblement énergétique $\left(40, \mathrm{E} \mathrm{m}{ }^{2} \mathrm{~s}{ }^{1}\right)$ est appliqué. L'éclairement est de $430,\left[\mathrm{E} \mathrm{m}^{-2} \mathrm{~s}^{-1}\right.$ lorsqu'il est dispensé par des lampes à halogénures métalliques (OSRAM H.Q.I., 3.5 Kw).

\subsection{Méthodes}

Des mesures hebdomadaires sont effectuées, portant sur la hauteur totale et le nombre de bourgeons débourrés (morphologiquement en activité) par rapport au nombre total de bourgeons morphologiquement visibles. L'allongement est exprimé en accroissement relatif $\left(L-L_{0} / L_{0} \times 100\right.$ où $L=$ longueur du rameau mesuré et $L_{0}$ la longueur initiale du rameau). Au temps $t_{w}$ des observations, tous les bourgeons des plants étaient sous forme écailleuse. 


\section{Résultats}

\subsection{Influence de la photopériode et de l'intensité de l'éclairement}

\subsection{Sur des boutures d'un an}

Par rapport à une photopériode courte $(9 \mathrm{~h})$ un éclairement de $16 \mathrm{~h}$ stimule fortement le débourrrement des bourgeons (fig. 1). Dès la troisième semaine de séjour dans ces conditions, plus de 20 p. 100 des bourgeons débourrent. C'est à la cinquième semaine que le maximum de débourrement est atteint ( 50 p. 100). Ensuite, la courbe s'abaisse indiquant une entrée progressive en repos de la majorité des bourgeons.

En conditions de jours longs également de $16 \mathrm{~h}$ mais obtenues grâce à un appoint de lumière faiblement énergétique, l'importance du débourrement est comparable à celle observée en $16 \mathrm{~h}$ (fig. 1). On observe, cependant, un décalage dans le temps puisque c'est à la huitième semaine que le maximum de débourrement est atteint. Les courbes obtenues en $7 \mathrm{~h}+9 \mathrm{~h}$ et en $9 \mathrm{~h}$ (fig. 1) sont en phase, l'amplitude étant plus grande pour le traitement en jours longs.

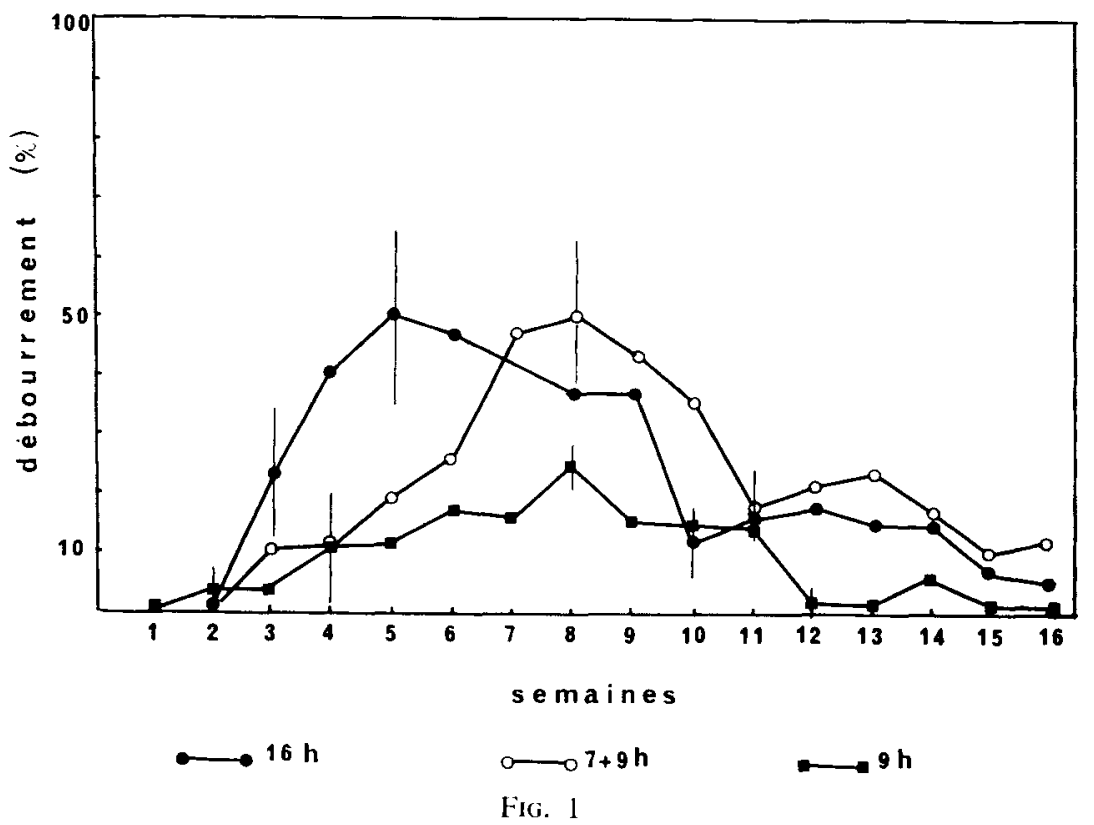

Influence de la photopériode sur le débourrement des bourgeons de jeunes Pseudotsuga menziesii.

Tous les bourgeons visibles de chaque plant sont considérés (5 plants par lot).

In/luence of photoperiodic treatments on the bud burst of young Pseudotsuga menziesii. All the morphological visible buds have been observed $(5$ plants each lot).

$16 \mathrm{~h}$ et $9 \mathrm{~h}$ : éclairement de $250 \mu \mathrm{E} \mathrm{m} \mathrm{m}^{-2} \mathrm{~s}^{\cdots-1}$.

$7+9 \mathrm{~h}$ : éclairement de $40 \mu \mathrm{E} \mathrm{m}{ }^{\circ}=\mathrm{s}$ 'suivi dun éclairement de $9 \mathrm{~h}$ de $250\left\lfloor\mathrm{E} \mathrm{E} \mathrm{m}^{-\cdots} \mathrm{s}^{-1}\right.$. 


\subsection{Sur des boutures de 4 ans}

A part la différence d'âge des boutures, cet essai est identique au précédent. Cependant, du fait du grand développement des plants, les résultats (fig. 2) sont exprimés en nombre de pousses en croissance, sans rapporter ce nombre à celui des bourgeons présents. Ces résultats confirment ceux obtenus précédemment : le débourrement et sa précocité sont fortement stimulés par une photopériode longue. De plus. un accroissement de l'intensité de l'éclairement toujours fourni pendant $16 \mathrm{~h}$ permet le débourrement quasi-immédiat d'un grand nombre de bourgeons. Un éclairement élevé permet à quelques bourgeons d'un plant placé en $9 \mathrm{~h}$ de débourrer après 4 semaines de traitement alors qu'en éclairement moins intense, il faut attendre 12 à 13 semaines pour obtenir le même résultat.

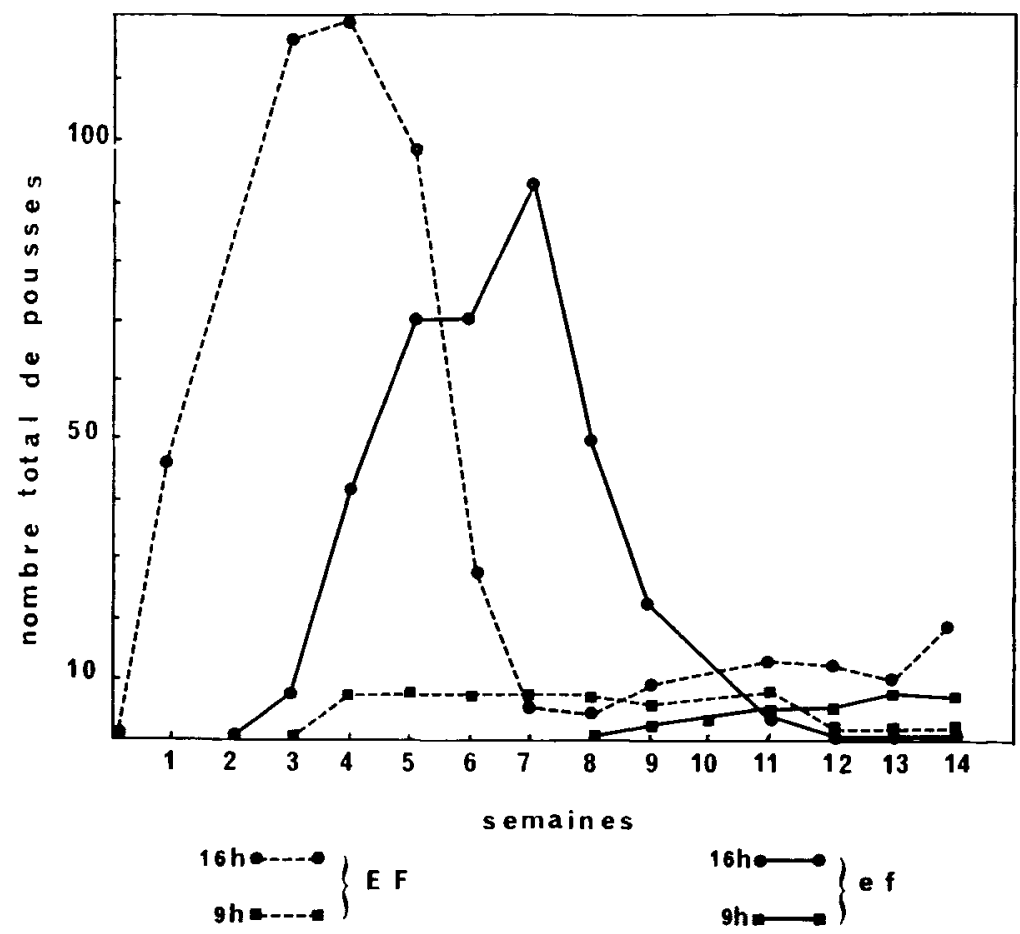

Fig. 2

Influence de l'intensité de l'éclairement donné durant 9 het $16 \mathrm{~h}$ sur le débourrement de bourgeons de Pseudotsuga menziesii agés de 4 ans.

I plant par condition. Tous les bourgeons en croissance sont considérés.

Influence of the intensity of lighting given during $9 h$ and $16 \mathrm{~h}$ on the hud burst of 4 years old Pseudotsuga menziesii. (I plant each condition). All the growing buds are numbered.

$\mathrm{EF}$ : éclairement de $430 \mu \mathrm{E} \mathrm{m} \mathrm{m}^{-\cdots} \mathrm{s}^{-1}$.

ef : ćclairement de $250 \mu \mathrm{E} \mathrm{m}^{-n} \mathrm{~s}^{\cdots}$.

On observe, enfin, dans ces conditions constantes, une phase d'arrêt de croissance marquée par la formation de bourgeons écailleux. Il semble qu'une seconde vague de 
croissance s'amorce pour la plante placée en $16 \mathrm{~h}$ d'éclairement intense (fig. 2). Quoiqu'il en soit, des plants issus de semis présentent également une réelle rythmicité de croissance lorsqu'ils reçoivent un éclairement continu $\left(9 \mathrm{~h}\right.$ à $250 \mathrm{fE} \mathrm{m}^{-2} \mathrm{~s}^{-1}$ et $15 \mathrm{~h}$ à $\left.40, \mathrm{Em} \mathrm{m}^{-2} \mathrm{~s}^{-1}\right)$. Ainsi, en 6 mois, 4 à 5 cycles végétatifs ont été observés sur les mêmes rameaux.

\subsection{Influence des températures basses}

Lorsque des plantes séjournent pendant des durées variables ( 3 semaines, 6 semaines et 9 semaines) à $+5^{\circ} \mathrm{C}$ et en jours courts avant d'être placées à $22^{\circ} \mathrm{C}$ en $16 \mathrm{~h}$ d'éclairement, on observe une inertie quasi-totale des bourgeons durant la période froide (fig. 3). Dès le transfert en conditions favorables à la croissance, le nombre de bourgeons qui rentrent en activité est d'autant plus important que les plants ont séjourné plus longtemps au froid. La vitesse dobtention du maximum de débourrement croît également. Enfin. le froid permet la croissance de tous les bourgeons d'un même arbre alors que, chez les plantes témoins non soumises au froid, seulement 65 p. 100 des bourgeons peuvent débourrer. Cette condition fait donc apparaître une hétérogénéité dans l'ensemble des bourgeons : certains, non dormants, pourraient croître

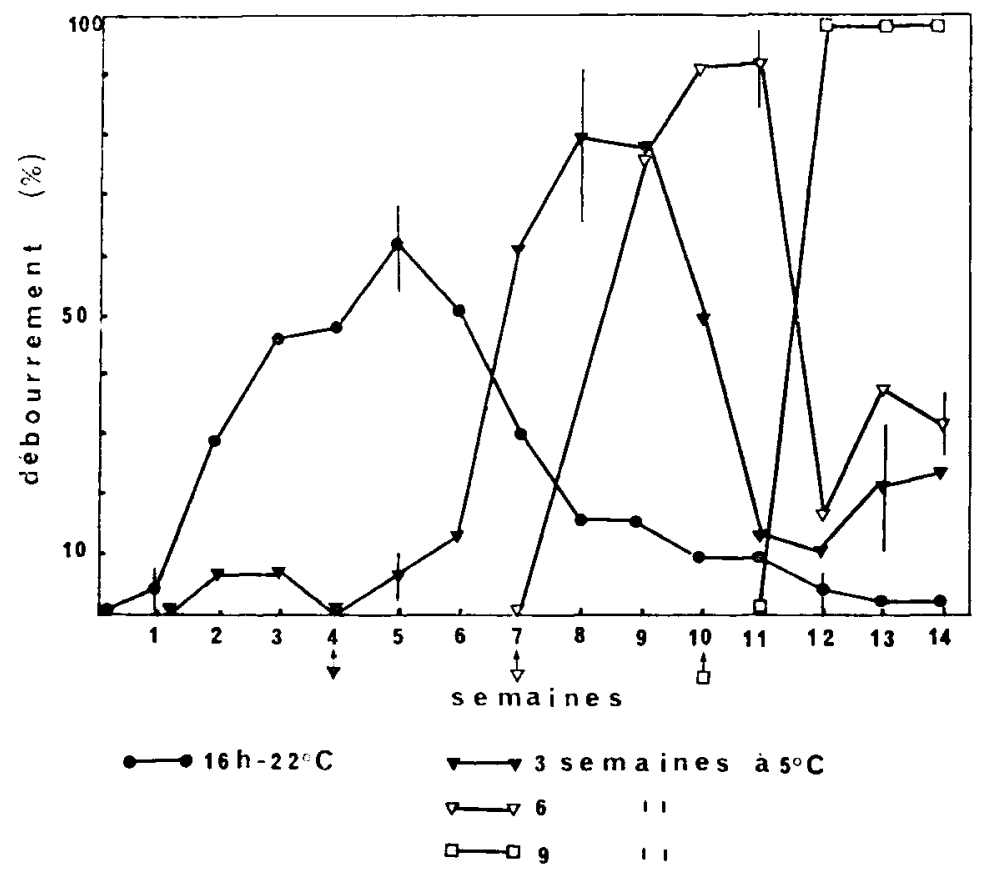

FIG. 3

Influence de la durée d'un traitement par le froid sur le débourrement des bourgeons. Tous les bourgeons visibles de chaque plant somt considérés (3 plants par lot).

Influence of the cold treament $\left(5^{\circ} \mathrm{C}\right)$ duration on the bud burst. All the morphological visible buds have been observed (3 plants each lot).

: Fin des 3 traitements par le froid.

: End of the 3 cold treatments. 
rapidement, d'autres, dormants, restant inertes. II ressort de ces observations que le froid amplifie et synchronise le débourrement observé en photopériode longue et à $22^{\circ} \mathrm{C}$.

L'allongement des rameaux dont la rythmicité est nettement marquée (fig. 4) est également fortement accru par le traitement par le froid. Si l'on compare la première phase d'allongement consécutive au débourrement soit spontané soit après action du froid, on observe que l'accroissement relatif des rameaux est fortement stimulé et ce, d'autant plus que la durée de froid est plus importante (fig. 4). La vitesse d'allongement est également augmentée.

\subsection{Influence de températures élevées}

Afin de savoir si des températures élevées, voisines de celles d'un été chaud, pourraient être à l'origine d'une seconde vague de croissance, de jeunes plants ont été

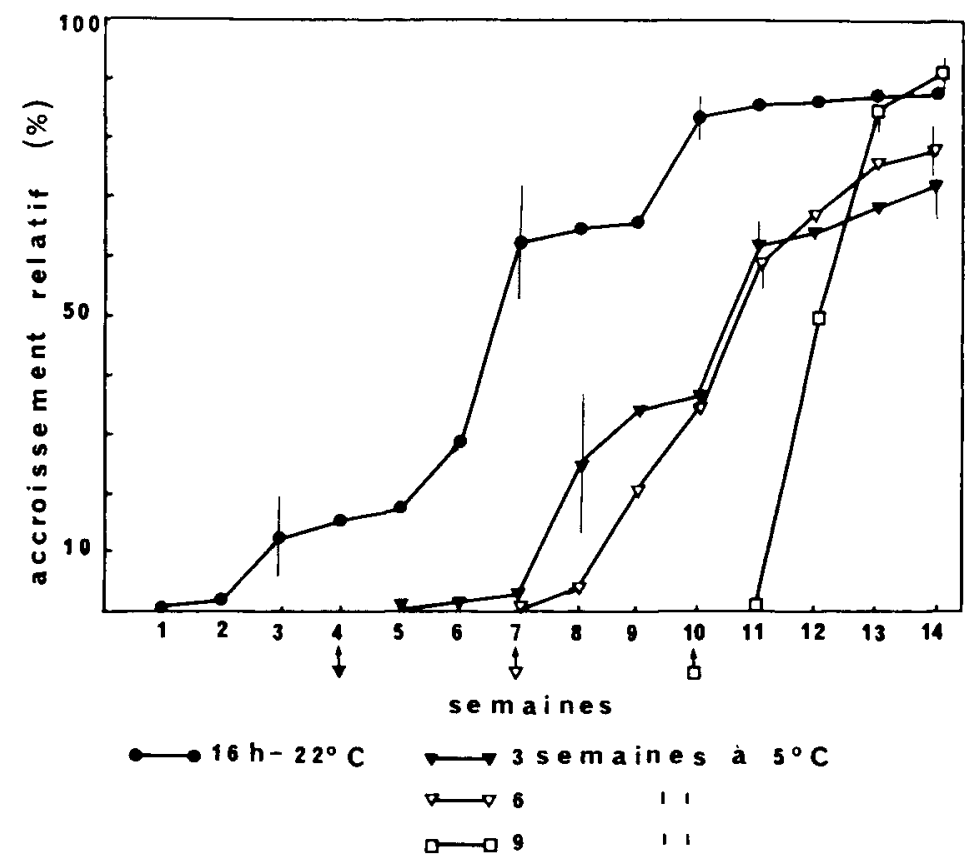

FIG. 4

Influence de la durée d'un traitement par le froid

sur laccroissement relatif des rameaux de jelmes Pseudotsuga menziesii.

3 à 5 rameaux sont sélectionnés par plant (rameaux supérieurs, moỵens et inférieurs) (3 plants par lot).

Influence of the cold treatment $\left(5^{\circ}\right)$

duration on the relative growth of shoots of Pseudotsuga menziesii.

3 to 5 shoots per plant have been selected (superior, middle and basal position).

: Fin des 3 traitements par le froid.

End of the 5 cold treatments. 


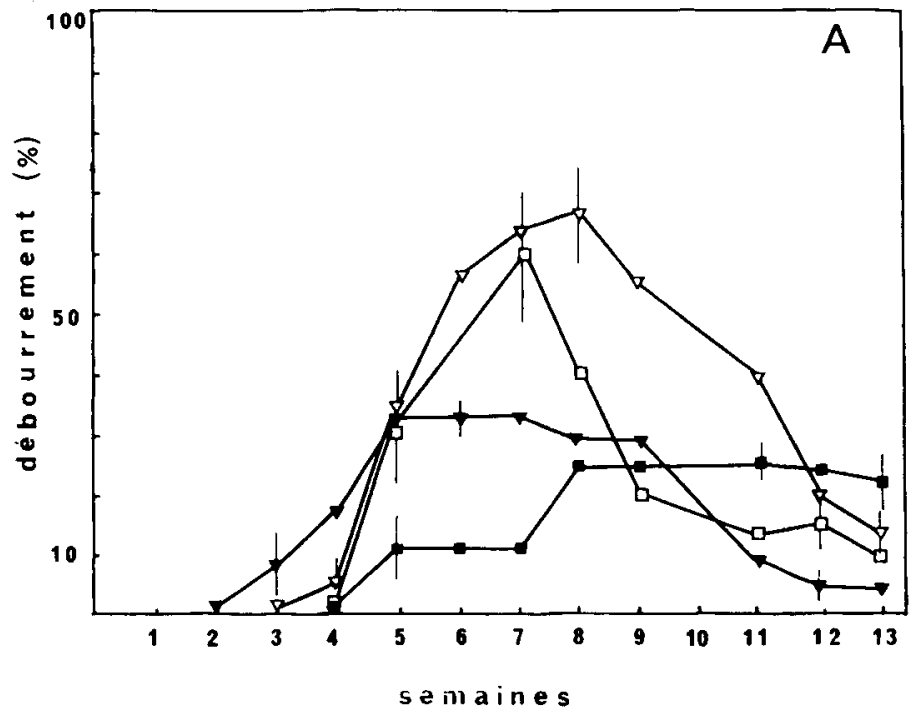

$\longrightarrow 9 \mathrm{~h}-22^{\circ} \mathrm{C} \quad \longrightarrow 1$ semaine à $35-25^{\circ} \mathrm{C}$

$\checkmark 2$ semaines

$\underset{0}{0} 3 \quad 11$

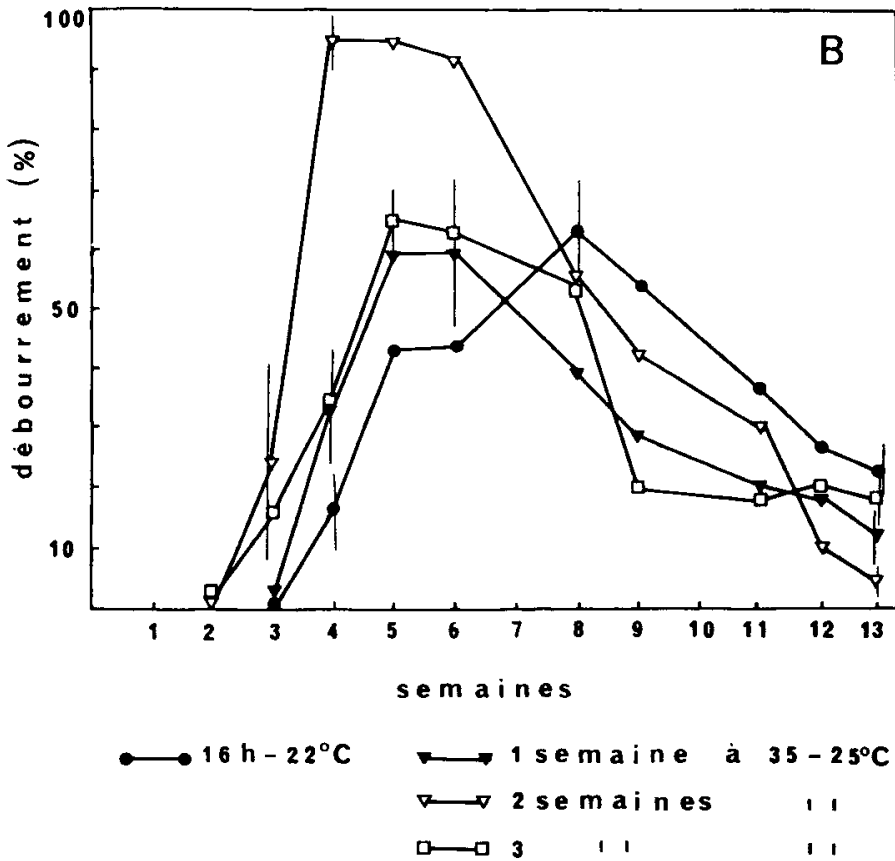

FIG. 5

Influence de températures élevées

sur le débourrement des bourgeons de jeunes plants de Pseudotsuga menziesii.

Tous les bourgeons visibles de chaque plant sont considérés (4 plants par lot).

Influence of hot temperatures on the bud burst of young Pseudotsuga menziesii plants.

All the morphological visible buds have been observed (4 plants each lot).

$5 \mathrm{~A}=$ en $9 \mathrm{~h}$.

$5 \mathrm{~B}=$ en $16 \mathrm{~h}$. 
placés à températures élevées. Les plants passent progressivement ( 2 semaines) de $12^{\circ} \mathrm{C}$ à $35^{\circ} \mathrm{C}$ (pendant $9 \mathrm{~h}$ ) et $25^{\circ} \mathrm{C}$ (pendant $15 \mathrm{~h}$ ). Deux photopériodes sont utilisées : $9 \mathrm{~h}$ et $16 \mathrm{~h}$. Des 3 durées de traitement par les températures élevées utilisées (1,2 et 3 semaines) c'est la durée moyenne qui produit les réactions les plus nettes aussi bien en $9 \mathrm{~h}$ qu'en $16 \mathrm{~h}$. Les pourcentages de débourrement sont, en effet, notablement accrus (fig. $5 \mathrm{~A}$ et $\mathrm{B}$ ). Ce type de traitement synchronise également la réaction des bourgeons : 2 semaines après le début du traitement tous les bourgeons sont actifs alors que c'est seulement après 6 semaines que le maximum de réactivité (65 p. 100) est observé chez les plantes témoins. La comparaison des figures $5 \mathrm{~A}$ et $5 \mathrm{~B}$ montre, à nouveau, l'effet favorable des jours longs sur le débourrement. L'interaction jours longs-températures élevées pendant 2 semaines se révèle très favorable à la réactivation de bourgeons écailleux.

\section{Discussion}

Les résultats présentés mettent l'accent sur le rôle positif sur la croissance des bourgeons du Pseudotsuga menziesii exercé par plusieurs facteurs du milieu : les températures fraîches et élevées d'une part, les photopériodes longues et des valeurs élevées d'éclairement, d'autre part. Bien que nous nous soyions attachés à n'utiliser des plants ne portant, en début d'expérience, que des bourgeons écailleux morpho. logiquement inactifs, nous ne pouvons affirmer que ces bourgeons étaient dormants. De plus, certains facteurs (température froide : fig. 3 et température chaude : fig. 5) provoquent une réaction de croissance des plants généralisée à l'ensemble des bourgeons alors que, chez les plants non traités, les réponses ne sont pas homogènes. La population de bourgeons était done en début d'expérience, physiologiquement hétérogène. Cette hétérogénéité a été reconnue chez d'autres végétaux ligneux (ChampaGnat. 1983 b).

On sait également que l'absence de réaction d'un bourgeon peut correspondre soit à des états dormants d'intensité différente soit à des inhibitions corrélatives (CrABBE, 1984). Il semble, en fait, que dormance sensu stricto et phénomènes corrélatifs interfèrent largement dans l'établissement du degré de réactivité d'un bourgeon (Champagnat, 1983 b ; Crabbe, 1984). Aussi, nos résultats se réfèrent-ils à l'action de différents facteurs sur le débourrement de bourgeons dont la majorité d'entre eux n'étaient pas dormants ou dont la dormance était faible. Il est probable, cependant, comme le montre l'hétérogénéité des réponses à certaines conditions expérimentales, que certains bourgeons présentaient une dormance plus profonde. Le rôle des températures basses et élevées ainsi que celui d'un fort éclairement étant alors, dans cette hypothèse, de lever les dernières limitations internes à la croissance. Ces limitations peuvent être aussi dues à une phase de post-dormance, idée classiquement admise (Champagnat, $1983 \mathrm{~b}$ ). Selon cet auteur rapportant des travaux effectués chez le noyer et chez le pêcher, cette post-dormance serait levée par des températures voisines de $15^{\circ} \mathrm{C}$. On peut également se référer à la définition de la quiescence (ROMBERGER, 1963) pour expliquer les différences de réactivité observées chez le Pseudotsuga menziesii, un bourgeon quiescent n'exigeant pas de températures basses pour lever sa dormance, mais réclamant seulement des températures plus élevées favorables à la croissance. De plus, la croissance est plus rapide lorsque la température est plus élevée (CAmpbell \& Sugano, 1978). Ces réactions ne sont observées qu'après que le 
besoin en froid ait été satisfait (Ritchie, 1984). Selon cet auteur, de jeunes Pseudotsuga menziesii débourrent à température moyenne, voisine de $20^{\circ} \mathrm{C}$, et en $16 \mathrm{~h}$ d'éclairement en une trentaine de jours à condition d'avoir reçu préalablement environ 1500 heures de froid. Dans les conditions optimales de croissance de nos expériences, c'est 14 jours après le passage au tiède (fig. 3) que tous les bourgeons débourrent à condition d'avoir reçu également environ 1500 heures de froid. La similitude des résultats de Ritchie (1984) et des nôtres pourrait laisser croire que, dans les deux cas, les jeunes plants étaient dormants. Nous avons observé que tel n'était pas le cas si l'on considère l'ensemble des bourgeons. Nous pouvons donc conclure, comme plus haut, à l'hétérogénéité physiologique du matériel mis en expérience. Certains bourgeons ont un besoin important en froid pour débourrer, d'autres ne présentent aucune exigence en réfrigération.

Lorsque la température est élevée $\left(35^{\circ} \mathrm{C}-25^{\circ} \mathrm{C}\right)$ ou que la photopériode est longue $(16 \mathrm{~h})$, de nombreux bourgeons débourrent. Non exigeants en froid, on ne peut les considérer comme dormants (ROMBERGER, 1963). On pourrait alors proposer l'hypothèse suivante : dans les cas de croissance estivale aboutissant à deux cycles végétatifs par an (pousses d'août). les bourgeons sont en état de dormance peu profonde levée par des étés particulièrement chauds, comme le suggère la figure 5. Encore faut-il qu'une hydratation suffisante des plants permette la reprise de croissance (BonNet-Masimbert \& LanARES, 1978).

Après une période de croissance plus ou moins prolongée, les bourgeons de Pseudotsuga menziesii vont différencier des écailles et les rameaux vont arrêter leur élongation. Cette séquence se produit en toutes conditions d'environnement permettant la croissance. Seules les caractéristiques de ce rythme vont différer selon l'environnement. Comme l'arrêt de croissance se manifeste en photopériodes longues et courtes, on ne peut impliquer de façon exclusive un de ces facteurs dans linduction de ce phénomène. Il est, cependant, beaucoup plus marqué en jours courts qu'en jours longs. Certaines interactions photopériode-température permettent, en effet, une reprise de croissance rapide. Ainsi, l'association $22^{\circ} \mathrm{C}-(9 \mathrm{~h}+15 \mathrm{~h})$ assure à la plante une croissance quasi-continuc marquée de paliers de courte durée. Ce ralentissement est accompagné de la formation de quelques écailles. Ces observations confirment le rôle joué par le milieu qui se traduit par la modulation de propriétés internes, comme LAVARENNE (1969) l'a clairement montré chez le chêne. NiTSCH (1957) avait déjà observé que les jours longs retardaient l'arrêt de croissance chez le Picea abies, mais pas définitivement. De même, Vince-Prue (1984) a observé que des interruptions de la nuit par des éclairements rouge clair et rouge sombre étaient plus efficaces que le seul rouge clair pour retarder la dormance chez Picea abies. II semble, en fait, en conditions contrôlées de Phytotron, que l'arrêt de croissance soit une conséquence de la phase de croissance préalable. Ce rythme résulterait d'une incompatibilité entre élongation et organogenèse comme CHAMPAGNAT (1983 a) le suggère. Il est très probable que l'arrêt de croissance et la différenciation des écailles qui l'accompagne sont l'expression de phénomènes complexes de corrélations faisant intervenir plusieurs organes, tels que : bourgeons, racines, feuilles et axe caulinaire (Champagnat, 1983 a ; Crabbe, 1984). Cette étude, déjà bien développée chez des ligneux feuillus (Champagnat, $1983 \mathrm{a}, \mathrm{b}$; CrabBe, 1984) pourrait également être entreprise chez le Pseudotsuga menziesii. De même, l'utilisation de la technique de boutures de nœuds, répandue chez les ligneux feuillus (ChAmpaGnat et al., 1971 ; Mauget \& Germain, 1980) gagnerait à être étendue au Pseudotsuga menziesii. Ainsi, l'hétérogénéité observée dans l'état physiologique des bourgeons d'un jeune arbre 
pourrait être décrite en soustrayant les bourgeons à la majeure partie des influences corrélatives. L'influence de la lumière (périodicité, qualité) sur les rythmes d'activité des bourgeons mériterait également d'être étudiée. Une recherche de marqueurs biochimiques, par exemple la mesure de l'équilibre hormonal, pourrait être réalisée dans des conditions permettant la comparaison des données physiologiques et biochimiques, contribuant ainsi à préciser la réalité physiologique des définitions comme CHAMPAGNAT (1983 b) en souligne l'intérêt.

\author{
Reçu le 6 janvier 1986. \\ Accepté le 2 mai 1986.
}

\author{
Summary \\ The behaviour of Pseudotsuga menziesii (Mirb.) Franco buds grown \\ under controlled conditions
}

The aim of this article was to describe and to measure the characteristics of the bud burst of 9 to 12 month old cuttings of Pseudotsuga menziesii as a function of variations in photoperiod, light energy and temperature. At the beginning of the experiments, all the buds were seemingly in a rest state. Compared with a short photoperiod $(9$ h) a $16 \mathrm{~h}$ lighting period $\left(250 \mu \mathrm{E} \mathrm{m}-\mathrm{s}^{-1}\right)$ accelerated and strongly stimulated the intensity of bud burst (fig. 1). The same result, although less intense, was observed when weak lighting $\left(40 \mu \mathrm{E} \mathrm{m}^{-2} \mathrm{~s}^{-1}\right)$ was given as a complement to the $9 \mathrm{~h}$ period (fig. 1). These results have been confirmed on 4 years old plants on which a high energy $\left(430 \mu \mathrm{E}^{-"} \mathrm{~s}^{-1}\right) 16 \mathrm{~h}$ lighting period strongly enhanced the number of reactivated buds (fig. 2) as compared with the reactivated buds numbered on plants placed under the same photoperiod but with less intense light $\left(250 \mu \mathrm{E} \mathrm{m}^{-2} \mathrm{~s}^{-1}\right)$. On those plants placed in $9 \mathrm{~h}$ photoperiod, only intense light slightly enhanced the bud burst.

A cold period $(5$ " $\mathrm{C}$ ) accelerated the growth of all buds of the cuttings if the treatment lasted 9 weeks. For shorter cold periods, the intensity of bud burst depended upon the duration of the stay at cold temperature (fig. 3). The elongation of the shoot of the first generation which occured immediately after the cold period was higher when the cold period was longer (fig. 4). Finally, high temperatures given alternatively $\left(35^{\circ} \mathrm{C}\right.$ during $9 \mathrm{~h}$ and $25^{\circ} \mathrm{C}$ during $15 \mathrm{~h}$ ) for 2 weeks enhanced appreciably the bud burst. 1 or 3 weeks of this treatment were less effective (fig. 5). These effects were obtained in non limiting conditions of water and mineral nutrition.

Whatever the culture conditions, a slow growth phase took place immediately after a rapid elongation period. This was morphologically visible by the formation of scaly buds or, in some cases, by a few scales surrounding green buds.

We discuss the physiological heterogeneity of the population of the buds of a cutting as revealed by their different photoperiodic or temperature needs for their bud burst. These factors are not essential for the growth of all the buds, but they synchronize and hasten the renewal of the growth. According to the results high temperatures associated with long summer days could explain the formation of lamma shoots by trees growing outside.

\title{
Références bibliographiques
}

Allen G.S., Owens J.N., 1972. The life history of Douglas fir. Environ. Can. For. Serv., FO 42. 4972, Ottawa, 138 p.

Barnola P., Champagnat P., Lavarenne S., 1977. Mise en évidence d'une dormance rythmique chez le Noisetier (Corylus avellana L.) cultivé en conditions contrôlées. C.R. Acad. Sci., Paris, série D, 284, 745-748. 